\title{
Toxic and Metabolic Myelopathies
}

\author{
Joana Ramalho, MD, ,"† Renato Hoffmann Nunes, MD,,", \\ Antonio José da Rocha, MD, PhD, ${ }^{\ddagger}$ and Mauricio Castillo, MD
}

\begin{abstract}
Myelopathy describes any neurologic deficit related to the spinal cord. It is most commonly caused by its compression by neoplasms, degenerative disc disease, trauma, or infection. Less common causes of myelopathy include spinal cord tumors, infection, inflammatory, neurodegenerative, vascular, toxic, and metabolic disorders. Conditions affecting the spinal cord must be recognized as early as possible to prevent progression that may lead to permanent disability. Biopsy is rarely performed, thus the diagnosis and management rely on patient's history, physical examination, laboratory results, and imaging findings. Here we review the clinical presentations, pathophysiological mechanisms, and magnetic resonance imaging findings of myelopathies related to metabolic or toxic etiologies.

Semin Ultrasound CT MRI 37:448-465 @ 2016 Elsevier Inc. All rights reserved.
\end{abstract}

\section{Introduction}

$\mathrm{M}$ yelopathy is a term used to describe neurologic deficits related to the spinal cord. It is usually compressive in nature, typically associated with neoplasm, degenerative disease, trauma, or infection, which may be managed surgically to relieve the cord compression in hopes of restoring normal function. Less common noncompressive myelopathies include inflammatory etiologies such as autoimmune, demyelinating, paraneoplastic, or infectious diseases and noninflammatory myelopathies, such as metabolic, toxic, or vascular disorders, which may not be amenable to surgical intervention and require different approaches to diagnosis and treatment.

Toxic and metabolic myelopathies may be further subdivided into (1) metabolic myelopathies associated with an identified nutrient deficiency including cobalamin or $\mathrm{B}_{12}$ vitamin, folate, copper, or vitamin E deficiencies; (2) toxic myelopathies associated with drugs and chemical agents such as heroin abuse, organophosphate poisoning, chemotherapeutic agents, and radiation therapy; (3) metabolic myelopathies with geographical predilection such as lathyrism and Konzo seen in India, Bangladesh, and Ethiopia and, (4) other rare

*Radiology Department, University of North Carolina Hospital, Chapel Hill, NC.

†Neuroradiology Department, Centro Hospitalar de Lisboa Central, Lisboa, Portugal.

¥Neuroradiology Division, Santa Casa de Misericórdia de São Paulo, Sao Paulo, Brazil.

Address reprint requests to Joana Ramalho, MD, Lg Sao Joao Baptista, N5 Apt105, 1600-760 Lisboa, Portugal. E-mail: joana-ramalho@netcabo.pt noninfectious and noninflammatory myelopathies including ketoacidosis myelopathy, spinal cord decompression illness, and surfer's myelopathy (Table 1). Toxic and metabolic myelopathies share clinical, electrophysiological, neuropathologic, and imaging features. Variable degrees of peripheral nerve and optic nerve involvement are present and usually known as a myeloneuropathy and preferential involvement of the dorsal columns and corticospinal tracts is seen. Our purpose is to review the uncommon toxic and metabolic myelopathies giving special emphasis to their clinical presentation, pathophysiological mechanisms and magnetic resonance imaging (MRI) findings.

\section{Myelopathies Associated With Nutrient Deficiencies}

\section{Cobalamin or $\mathrm{B}_{12}$ Vitamin Deficiencies Definition}

Vitamin $B_{12}$ deficiency causes myelopathy, neuropathy, and neuropsychiatric disturbances. In the spinal cord, it manifests as a subacute combined degeneration, characterized by degeneration of the lateral and posterior columns. It is considered the most common metabolic myelopathy. ${ }^{1-3}$

\section{Pathophysiology}

Vitamin $\mathrm{B}_{12}$ deficiency is due to inadequate intake and malabsorption. Inadequate intake is seen in strict vegetarians and is considered rare in the Western World. Pernicious anemia (the most common cause in the West), gastrectomy, 
Table 1 Toxic and Metabolic Myelopathies

Key Findings

Myelopathies associated with nutrient deficiency

Cobalamin or $\mathrm{B}_{12}$ vitamin deficiency

Subacute combined degeneration, peripheral neuropathy and neuropsychiatric disturbances. Megaloblastic anemia may be present.

Nitrous oxide toxicity (associated with $B_{12}$ inactivation)

Folate deficiency

Copper deficiency

Vitamin E deficiency

AIDS-associated myelopathy (mechanism not completely understood)

Previous exposure to $\mathrm{N}_{2} \mathrm{O}$ ("anesthesia paresthetica").

Similar to $B_{12}$ vitamin deficiency.

Subacute combined degeneration and history of gastric surgery.

Progressive spinocerebellar syndrome with lesions in the posterior columns on MRI.

Patients who are HIV positive presenting with lesions in the posterior columns predominantly in the upper thoracic cord on MRI.

Toxic myelopathies associated with drugs and chemical agents

Heroin myelopathy

Progressive involvement with selective involvement of the ventral pons and lateral and posterior columns of the spinal cord.

Hepatic myelopathy

Pure motor spastic paraparesis with involvement of the lateral corticospinal tracts. Evidence of manganese deposition in the basal ganglia (high signal on T1-WI) is common.

Organophosphate poisoning

Delayed myelopathy and neuropathy presenting with spinal cord atrophy that persists long after the cholinergic effects of organophosphate had subsided.

Chemotherapy and radiation therapyrelated myelopathies

History of chemotherapy and radiation therapy. The affected spinal cord segment is in the same area as the radiation-induced bone marrow changes.

Toxic myelopathies with geographical predilection

Spinal sea stroke

Subacute myelo-optico neuropathy (clioquinol toxicity)

Lathyrism (seen in India, Bangladesh, and Ethiopia) and Konzo (Africa)
Spinal cord infarct in patients swimming in the sea of specific locations in the globe.

Subacute myelo-optic neuropathy after clioquinol treatment.

Specific dietary habits.

Other noninfectious and noninflammatory myelopathies

Ketoacidosis myelopathy

Spinal cord decompression illness

Rare complication of diabetic ketoacidosis.

Patients diagnosed with decompression sickness presenting with patchy areas of increased T2 signal intensity in the spinal cord with no segmental distribution.

Surfer's myelopathy

Longitudinally extensive T2 hyperintense central cord lesion in the midthoracic region in novice surfers.

*All listed entities cause a very similar pattern of clinical and imaging involvement of the posterior and lateral columns.

intestinal infections and ileal abnormalities (Helicobacter pylori, Diphyllobothrium latum, human immunodeficiency virus, intestinal tuberculosis or lymphoma, celiac disease, Whipple's disease, inflammatory bowel disease, radiation enteritis, graft-versus-host disease, pancreatic disease, ZollingerEllison syndrome, and tropical sprue, bacterial overgrowth) lead to malabsorption ${ }^{1,4}$. Other conditions include elderly (related to atrophic gastritis-related hypochlorhydria induced food-Vitamin $\mathrm{B}_{12}$ malabsorption), hereditary enzymatic defects and mutations in genes encoding endocytic receptors involved in ileal absorption and cellular $\mathrm{Cbl}$ uptake (Mutations in CUBN and AMN (selective $\mathrm{Cbl}$ malabsorption and proteinuria: Imerslund-Gräsbeck syndrome), mutations in gastric intrinsic factor (GIF) or TCN2 (transcobalamin) leading to absence of the protein or presence of abnormal protein, disorders of intracellular processing and utilization of vitamin $\mathrm{B}_{12}$, disorders involving the synthesis of cobalamin cofactors (cblA to cblG), and medications (Antacids, H2 blockers, metformin, sunitinib).
Coenzyme vitamin $\mathrm{B}_{12}$ (cobalamin), which together with folate, is essential for the formation of methionine from homocysteine tetrahydrofolate. It is absorbed in the ileal microvilli after binding to the intrinsic factor. Tetrahydrofolate is necessary for normal DNA synthesis and methionine is converted to S-adenosylmethionine that is necessary for methylation of myelin sheath phospholipids. $\mathrm{B}_{12}$ deficiency results in decreased activity of cobalamin-dependent methylcobalamin esterase enzyme with resultant elevation of methylmalonic acid and reduced myelin basic protein methylation with fragile myelin that is susceptible to demyelination and vacuolization. ${ }^{1,4}$

\section{Clinical Presentations}

Vitamin $B_{12}$ deficiency may lead to serious neurologic complications including neuropathy, myelopathy, neuropsychiatric disturbances, and optic neuropathy. These may be accompanied by hematologic manifestations of cobalamin deficiency such as anemia, macrocytosis, neutrophil hypersegmentation, or megaloblastic marrow changes. Subacute 
Table 2 Clinical Manifestations of Vitamin $B_{12}$ Deficiency"

Neurologic manifestations (may be the earliest and often the only manifestation of $\mathrm{B}_{12}$ deficiency)

Myelopathy (subacute combined degeneration)

Numbness, weakness, and paresthesia of the extremities that primarily affect the lower extremities, often symmetric, and progresses in a distal-to-proximal manner. Later this myelopathy progresses to unsteady gait, poor coordination, sensory deficits, and bowel or bladder dysfunctions

Physical examination: signs of dorsal column involvement include loss of position and vibration sense or ataxia. Lateral column involvement includes spasticity, hyperreflexia, and positive Babinski sign. Involvement of the spinothalamic tracts with sensory level may also be present

Peripheral neuropathy and optic neuropathy may be seen

\section{Psychiatric manifestations \\ Cognitive impairment \\ Personality and mood changes with depression and psychosis}

\section{Hematologic manifestations}

Megaloblastic anemia or megaloblastic marrow changes

Pancytopenia (leukopenia and thrombocytopenia)

*Adapted from Kumar.

combined degeneration presents as a myelopathy (clinical manifestations are summarized in Table 2). ${ }^{1}$

\section{Pathology Findings}

Neuropathologic findings initially include swelling of myelin sheaths with little change in axons in the posterior columns of the upper cervical segments (funiculus gracilis and spinocerebellar tracts) and in the lateral columns, which typically commence in the upper thoracic cord but can extend to involve other levels. Later, myelin sheath and axonal degeneration and loss are seen leading to Wallerian-type degeneration of the long tracts which may extend symmetrically in the cranial-caudal direction. Other sites of involvement include peripheral nerves, cerebrum, and optic nerves. ${ }^{4}$

\section{Imaging Findings}

MRI shows increased signal intensity in the posterior and lateral white matter columns of spinal cord on T2-weighted images (T2-WI) (Fig. 1). The "inverted V" or "inverted rabbit ears" signs are often present and described as symmetrical T2 hyperintensities in the lateral aspects of the dorsal columns (Fig. 2). ${ }^{5}$ Cervical and upper thoracic cord are most commonly and severely affected as a continuous long segment. ${ }^{2,4,6,7}$ Mild cord expansion and contrast enhancement may be seen. ${ }^{8}$ Spinal cord lesions may resolve after appropriate therapy, however cord atrophy develops in some patients. ${ }^{9}$ Rarely, diffuse white matter abnormalities (supratentorial and infratentorial) suggestive of a leukoencephalopathy, particularly affecting the corticospinal tracts, may be seen. ${ }^{10,11}$

\section{Diagnosis}

Diagnosis is confirmed by low serum $B_{12}$ or if the $B_{12}$ is borderlined by elevated homocysteine and methylmalonic acid. Hematological changes such as megaloblastic anemia are not reliable markers of the disease. ${ }^{1}$

\section{Treatment or Prognosis}

Treatment goals are to reverse the signs and symptoms of deficiency, replete body stores, ascertain the cause of the deficiency and monitor response to therapy. Severe impaired absorption requires parenteral therapy and most common recommended treatment is with $\mathrm{B}_{12}$ intramuscular injections continued monthly for life. Spine and brain MRI findings may resolve within a few months after starting therapy and the degree of recovery is inversely proportional to symptom duration and severity. Therefore, early diagnosis leads to full recovery. ${ }^{1}$

\section{Nitrous Oxide Toxicity}

\section{Definition}

Nitrous oxide $\left(\mathrm{N}_{2} \mathrm{O}\right.$ or "laughing gas") is a commonly used inhalational anesthetic that causes irreversible inactivation of vitamin $B_{12}$ by oxidation and has been associated with a myeloneuropathy resembling subacute combined degeneration seen with $\mathrm{B}_{12}$ deficiency.

\section{Pathophysiology}

Methylcobalamine, the active form of intracellular vitamin $B_{12}$, is required by the methionine synthetase enzyme to convert homocysteine into methionine and methyltetrahydrofolate into tetrahydrofolate. $\mathrm{N}_{2} \mathrm{O}$ inactivates vitamin $\mathrm{B}_{12}$ by irreversible oxidation of the cobalt center of methylcobalamine from the monovalent to bivalent and trivalent forms. The methionine synthesis pathway is thereby inhibited. In normal subjects, blocking of methionine synthesis by $\mathrm{N}_{2} \mathrm{O}$ does not cause symptoms. However, subclinical vitamin $\mathrm{B}_{12}-$ deficient individuals are prone to neurologic degeneration because $\mathrm{N}_{2} \mathrm{O}$ causes exhaustion of the residual stocks of vitamin $\mathrm{B}_{12}{ }^{1,12}$

\section{Clinical Presentations}

Myeloneuropathy results from chronic exposure or after a single exposure in individuals with $\mathrm{B}_{12}$ deficiency. Signs and symptoms appear rapidly with $\mathrm{N}_{2} \mathrm{O}$ toxicity and may be seen despite a normal serum $\mathrm{B}_{12}$ level. Less commonly, symptoms may be delayed up to 2 months after acute exposure. Neurologic manifestations may include a myelopathy (subacute 

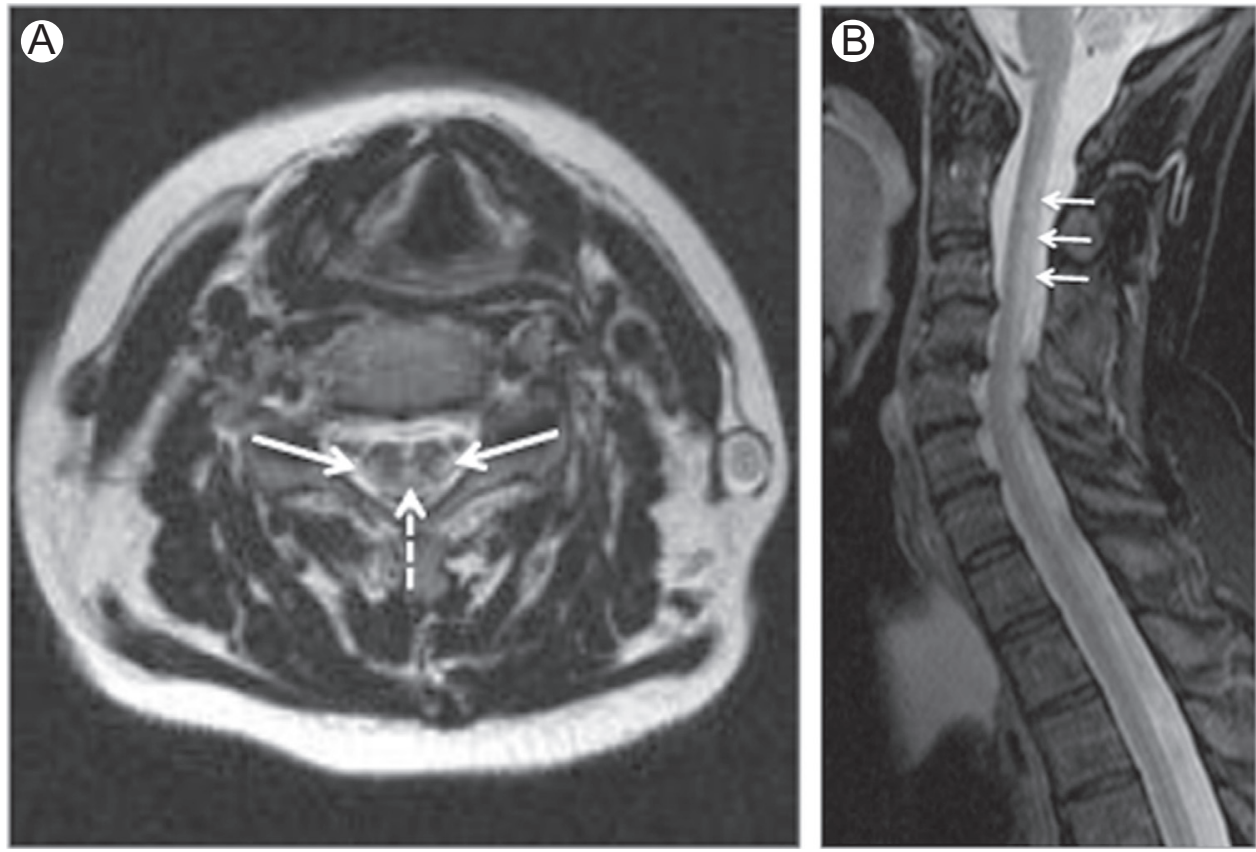

Figure 1 Vitamin $B_{12}$ deficiency. Axial (A) T2-WI in a patient with vitamin $B_{12}$ deficiency shows high-signal intensity involving the dorsal (dashed arrow) and lateral columns (white arrows), also seen on sagittal T2-WI (B) (arrows).

combined degeneration), neuropathy, myeloneuropathy, and mental status changes. ${ }^{1,12}$

\section{Pathology Findings}

Neuropathologically, the findings are similar to Vitamin $B_{12}$ related subacute spinal degeneration which are characterized by reversible swelling of myelin sheaths in the posterior and lateral columns. Later, myelin sheath and axonal degeneration are seen leading to irreversible Wallerian-type degeneration of the long tracts. ${ }^{12}$

\section{Imaging Findings}

MRI typically shows high T2 signal intensity in the posterior columns (sometimes in the lateral and anterior columns) of the cervical or thoracic spinal cord with often several vertebral bodies in length (Fig. 3). Modest expansion of the spinal cord may be seen. Contrast enhancement is rare and generally mild when it present. ${ }^{9,12,13}$

\section{Diagnosis}

The diagnosis of myeloneuropathy because of $\mathrm{N}_{2} \mathrm{O}$ is a clinical one and should be considered in patients who develop neurologic symptoms following surgical and dental procedures ("anesthesia paresthetica") or in recreational users.,

\section{Treatment or Prognosis}

Prevention includes prophylactic administration of vitamin $\mathrm{B}_{12}$ weeks before surgery in individuals with a borderline vitamin serum level who receives $\mathrm{N}_{2} \mathrm{O}$ anesthesia. ${ }^{.}$Treatment involves high doses of vitamin $\mathrm{B}_{12}$. Administration of methionine may also be required. Exogenous methionine provides a direct substrate for methionine synthetase whereas the body slowly replaces the inactive vitamin $B_{12}$ and starts repletion of
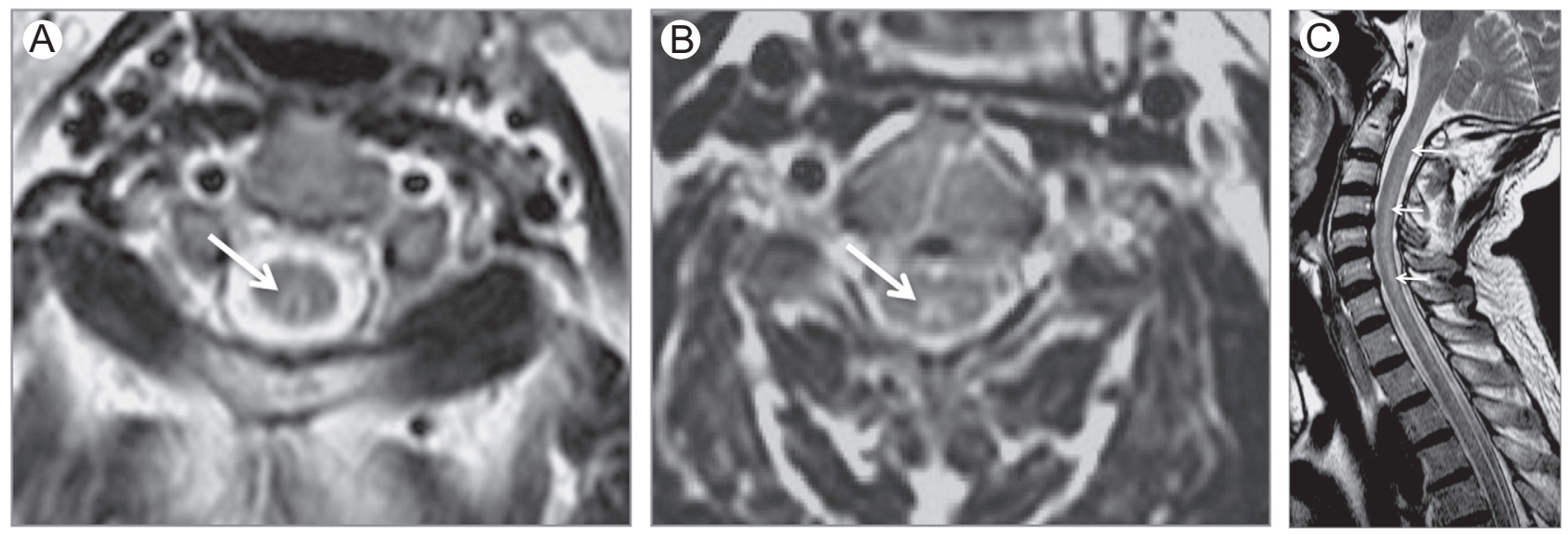

Figure 2 Vitamin $B_{12}$ deficiency. Axial (A and B) and sagittal (C) T2-WI in a patient with vitamin $B_{12}$ deficiency show highsignal intensity involving the dorsal columns (arrows). Note typical "inverted V" or "inverted rabbit ears" on axial images. 

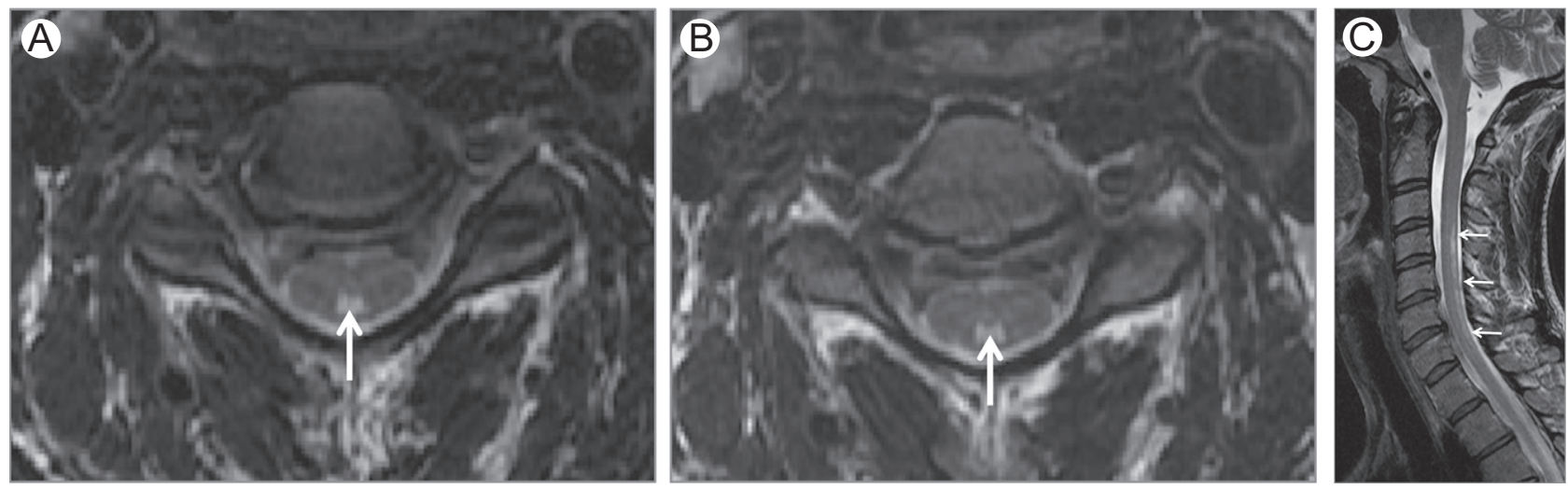

Figure 3 Nitrous oxide toxicity. Axial (A and B) and sagittal (C) T2-WI of the cervical spine in a patient with myelopathy after surgery with nitrous oxide anesthesia. Imaging findings are typical of vitamin $\mathrm{B}_{12}$ deficiency myelopathy with high $\mathrm{T} 2$ WI signal involving the dorsal columns (arrows). This patient had undiagnosed $\mathrm{B}_{12}$ deficiency and nitrous oxide administration resulted in subacute combine degeneration.

endogenous methionine. Improvement of symptoms and MRI changes are seen after replacement therapy is started. ${ }^{12}$

\section{Folate Deficiency \\ Definition}

Folate deficiency rarely exists in a pure state and is often associated with conditions that affect other nutrients. The most common manifestation of folate deficiency is a myelopathy or myeloneuropathy resembling subacute combined degeneration seen with $\mathrm{B}_{12}$ deficiency.

\section{Pathophysiology}

Folate is a water-soluble B vitamin present in fresh green vegetables, citrus fruits, legumes, and liver. As with $B_{12}$, folate acts as an important cofactor in DNA synthesis and methylation pathways. Its deficiency results from reduced intake of folate-rich products, impaired absorption, or increased folate metabolism. It is associated with alcoholism, small bowel disorders, and drugs such as methotrexate and trimethoprim. Folate absorption may be decreased with reduced gastric secretions in patients after gastric surgery, atrophic gastritis, acid-suppressive therapy, and acid neutralization by treatment of pancreatic insufficiency. ${ }^{1,14}$

\section{Clinical Presentations}

Folate deficiency causes similar manifestations to those of vitamin $B_{12}$ deficiency because of its importance in production of methionine and tetrahydrofolate. For unclear reasons, neurologic and hematologic manifestations due to folate deficiency are rare, mild, and confounding. Neurologic manifestations of folate deficiency include a myelopathy, peripheral neuropathy, optic neuropathy, and cognitive or behavioral manifestations. ${ }^{1,14}$

\section{Pathology Findings}

Pathologically, folate deficiency myelopathy is identical to vitamin $B_{12}$ deficiency myelopathy and characterized by reversible swelling of myelin sheaths in the posterior and lateral columns. ${ }^{1,4,10,14}$

\section{Imaging Findings}

Subacute combined degeneration due to folate deficiency resembles other forms of subacute combined degeneration (Fig. 4). 1,4,9,14

\section{Diagnosis}

Attribution of neurologic manifestations to pure folate deficiency requires exclusion of other causes. Serum folate levels between 2.5 and $5 \mu \mathrm{g} / \mathrm{L}$ may be indicative of a mildly compromised folate status. Erythrocyte folate is more reliable than plasma folate because its levels are less affected by shortterm fluctuations in intake. Plasma homocysteine levels are typically elevated in patients with clinically significant folate deficiency. ${ }^{1,14}$

\section{Treatment or Prognosis}

Daily doses, as high as $20 \mathrm{mg}$, may be necessary in patients with malabsorption. Reduced folates are required only when folate metabolism is impaired by drugs such as methotrexate or inborn errors of metabolism. Plasma homocysteine is best for monitoring response to therapy as it decreases within a few days of instituting folate therapy but does not respond to inappropriate vitamin $\mathrm{B}_{12}$ therapy. ${ }^{1,14}$

\section{Copper Deficiency Definition}

Acquired copper deficiency may cause myelopathy or myeloneuropathy that resembles subacute combined degeneration. ${ }^{1,15}$

\section{Pathophysiology}

Copper permits electron transfer in key enzymatic pathways. It is a component of metalloenzymes, including cytochrome-c oxidase, that have a critical role in the structure and function of the nervous system. Reduction in cytochrome-c oxidase activity may be the basis for neurologic dysfunction in copper deficiency. ${ }^{16}$ Because of copper's ubiquitous distribution and low daily requirement, acquired dietary copper deficiency is rare. The coexistence of multiple causes of copper deficiency 

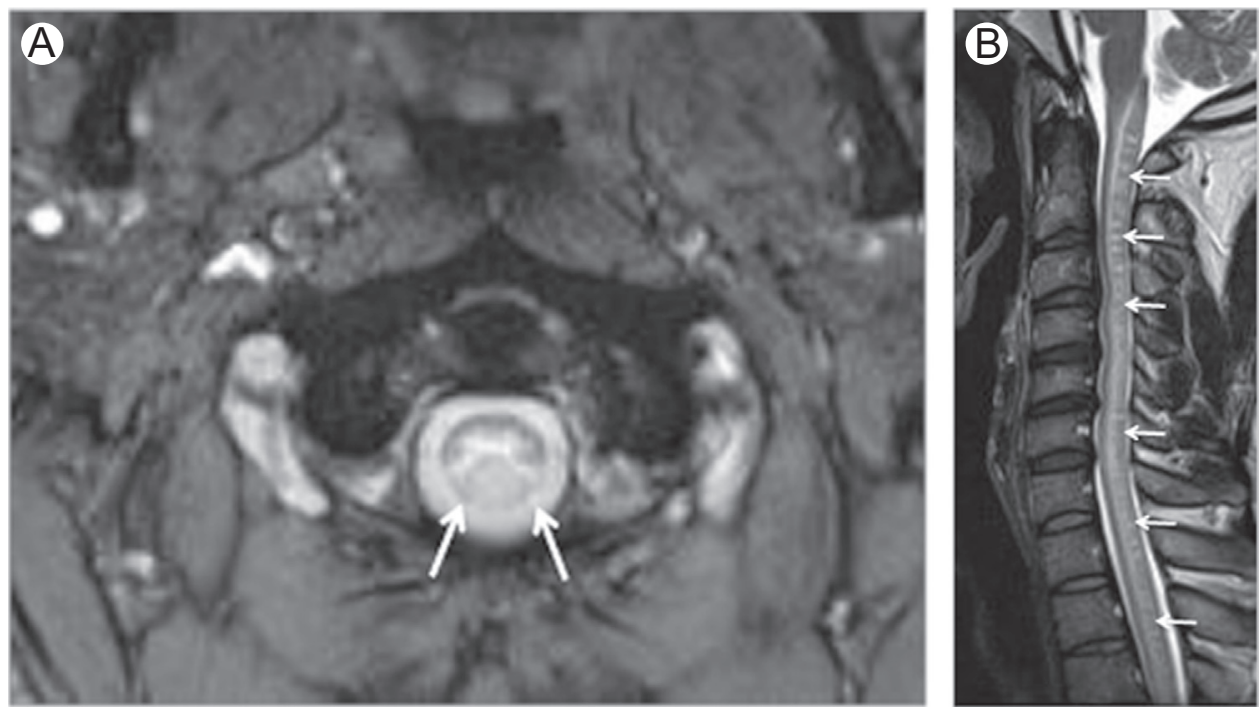

Figure 4 Folate deficiency. Axial (A) and sagittal (B) T2-WI in a patient with folate deficiency show high-signal intensity involving the dorsal columns (arrows).

increases the chances of development of a clinically deficiency state. The most common cause of acquired copper deficiency is a history of gastric surgery. Typically, neurologic manifestations are delayed by years following gastric surgery. Excessive zinc ingestion is also a recognized cause of copper deficiency. Additionally, copper deficiency has been reported in enteropathies associated with malabsorption such as cystic fibrosis, sprue, bacterial overgrowth, and inflammatory bowel disease. ${ }^{1}$

\section{Clinical Presentations}

Neurologic manifestations are similar to those seen with $B_{12}$ deficiency with which they may coexist. The most common manifestation is that of a myelopathy presenting with a spastic gait and prominent sensory ataxia because of dorsal column dysfunction. Central nervous system (CNS) demyelination, peripheral neuropathy, and optic neuritis also occur. ${ }^{1,16}$

\section{Pathology Findings}

Copper deficiency-associated myelopathy has been well described in animal models. The typical distribution of spinal cord lesions is that of greater involvement of the cervical cord with less severe changes in the thoracic and lumbar segments. Wallerian degeneration and demyelination with microcavitation of the white matter of the spinal cord, particularly affecting the dorsal columns and brainstem, are typical. ${ }^{16}$

\section{Imaging Findings}

The most common abnormality on the spine MRI is increased T2-WI signal in the dorsal columns, mostly in the cervical cord; contrast enhancement is not seen (Fig. 5). Additionally, signal changes involving the lateral columns and extension of the pyramidal tract signal changes into the brainstem have been reported. These findings are similar to those seen in patients with vitamin $\mathrm{B}_{12}$ deficiency. ${ }^{1,15-18}$

\section{Diagnosis}

Laboratory indicators of copper deficiency include a decrease in serum copper or ceruloplasmin and in 24-hour urinary copper excretion. Changes in serum copper usually parallel ceruloplasmin concentration. Serum zinc and 24-hour urinary zinc excretion levels should be obtained and an elevation in these should prompt an aggressive search for an exogenous source of zinc. ${ }^{1,16}$

\section{Treatment or Prognosis}

Oral copper supplement is the method of supplementation. Response of hematological parameters (including bone marrow findings) is prompt and often complete. Recovery of neurologic signs and symptoms is variable. In patients in whom excess zinc ingestion is the cause, stopping zinc supplements may suffice and no additional copper supplements may be required. ${ }^{1,15,18}$

\section{Vitamin E Deficiency \\ Definition}

Vitamin E deficiency manifests as a progressive spinocerebellar syndrome.

\section{Pathophysiology}

Vitamin $\mathrm{E}$ is as a fat-soluble antioxidant preventing peroxidation of membrane fatty acids. Most vitamin $\mathrm{E}$ in the human body is localized in adipose tissues. More than 2 years are required for adipose tissues to reach steady-state levels in response to changes in dietary intake. In adults, vitamin $\mathrm{E}$ deficiency is most often associated with fat malabsorption syndromes (celiac disease, cystic fibrosis, and cholestasis) and other intestinal disorders. In children, vitamin E deficiency may be seen because of genetic in alphatocopherol transfer protein, abetalipoproteinemia, or defects in chylomicron synthesis and secretion. 

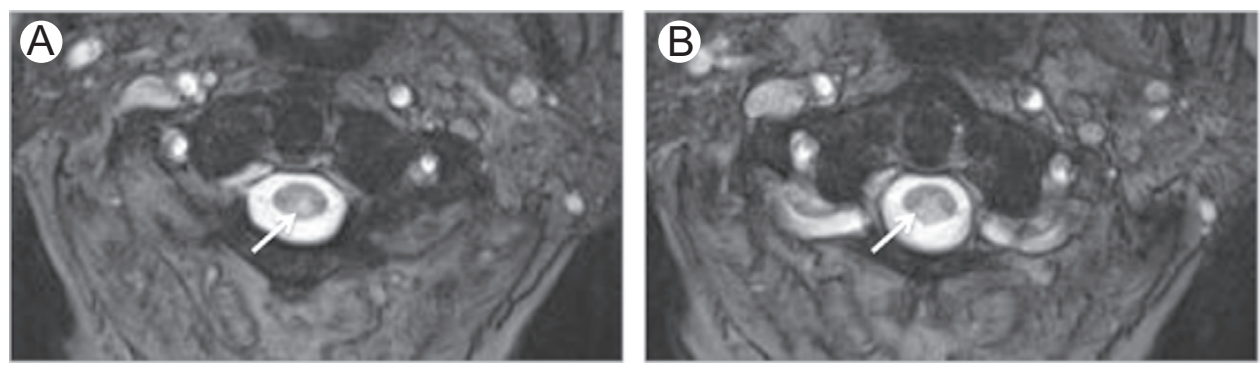

Figure 5 Copper deficiency. (A and B) Axial T2-WI at C1-2 level in a patient with Cu deficiency demonstrate high-signal intensity involving dorsal columns (arrows).

\section{Clinical Presentations}

Neurologic manifestations of vitamin E deficiency include a progressive spinocerebellar syndrome, similar to those of Friedreich ataxia, and a peripheral neuropathy with gait difficulty, hyporeflexia or areflexia, pyramidal signs, impaired position and vibration perception, and dysarthria. Pigmented retinopathy, myopathy, movement disorders, and gaze palsies have also been reported. ${ }^{1,19}$

\section{Pathology Findings}

Lesions in the dorsal root ganglia, posterior medullary tracts, cerebellar cortex, spinocerebellar tracts, anterior horn cells, and peripheral nerves have been reported. ${ }^{20}$

\section{Imaging Findings}

MRI may show high-signal lesions on T2-WI in the posterior columns. Cerebellar atrophy may be present. ${ }^{21,22}$

\section{Diagnosis}

Serum vitamin E levels are dependent on concentrations of serum lipids. Hyperlipidemia or hypolipidemia can increase or decrease serum vitamin $E$ without reflecting similar alterations in tissue levels. Effective serum vitamin E levels are calculated by dividing the serum vitamin $\mathrm{E}$ by the sum of serum cholesterol and triglycerides. In patients with neurologic manifestations serum vitamin $\mathrm{E}$ levels are frequently undetectable. $^{1}$

\section{Treatment or Prognosis}

Replacement of vitamin E is treatment of choice. Improvement of neurologic symptoms or signs in severe cases may not occur. ${ }^{1}$

\section{AIDS-Associated Myelopathy Definition}

AIDS-associated myelopathy, also called vacuolar myelopathy, is a spinal cord disease in human immunodeficiency virus (HIV) positive patients. Clinical manifestations, imaging findings, and histopathology resemble that of subacute combined degeneration because of vitamin $B_{12}$ deficiency. It may occur at any stage during the course of HIV infection but is more common with advanced immunosuppression. Reliable data on the incidence and prevalence of myelopathy in HIV patients are not available. ${ }^{1,23}$

\section{Pathophysiology}

The cause of vacuolar myelopathy is unknown. Increased prevalence of $\mathrm{B}_{12}$ deficiency occurs in HIV patients with neurologic symptoms but its significance is unclear. Impairment in methylation pathways related or not to viral infection of the spinal cord have been proposed. ${ }^{1,23,24}$

\section{Clinical Presentations}

Manifestations of vacuolar myelopathy are varied and often include painless spastic paraparesis that is slowly progressive with a sensory ataxia. There may be an associated sphincter and erectile dysfunctions. Overlapping peripheral neuropathy may confound the diagnosis. Hyperreflexia at the knees with reduced ankle reflexes are common. ${ }^{23}$

\section{Pathology Findings}

Vacuolar myelopathy is characterized by intralamellar vacuolation in the spinal white matter throughout the lateral and posterior columns of the thoracic and cervical segments. ${ }^{23,25}$

\section{Imaging Findings}

MRI features of vacuolar myelopathy include high T2 intensity lesions in the posterior columns predominantly in the upper thoracic cord. Frequently, the spinal cord may be normal or show atrophy and nonspecific patchy T2-WI abnormalities. ${ }^{23,25}$

\section{Diagnosis}

Diagnosis is made by exclusion by ruling out vitamin $B_{12}$ deficiency, human T-lymphotrophic virus-1, and neurosyphilis. Cerebrospinal fluid (CSF) may be normal or show mild pleocytosis with mild protein elevation. Similar findings may be seen in asymptomatic HIV patients.

\section{Treatment or Prognosis}

There is no effective therapy for HIV-associated myelopathy. The role of antiretroviral therapy is not established, however following antiretroviral therapy MRI may show significant reduction of the T2 hyperintensity of the posterior columns. ${ }^{23,25}$ 


\section{Toxic Myelopathies Associated With Drugs and Chemical Agents}

\section{Heroin Myelopathy \\ Definition}

Myelopathy induced by heroin is progressive with selective involvement of the ventral pons and lateral and posterior columns of the spinal cord and may be associated with inhalation ("chasing the dragon") or intravenous use. 1,26,27

\section{Pathophysiology}

Proposed mechanisms include direct toxicity, vasculitis, or hypersensitivity reaction. Isolated toxicity seems less probable as systemic toxins typically involve the nervous system more diffusely along with other organs. Generalized vasculitis would be unlikely to first affect the spinal cord. ${ }^{27}$ Thus, hypersensitivity is the predominant explanation as the myelopathy may occur after a period of abstinence followed by a single use. ${ }^{1,26-30}$ Hypotension may possibly result in border-zone infarctions in the cord. ${ }^{1}$ However, hypotension is unlikely to be severe enough to damage the spinal cord and spare the more ischemia-sensitive neurons of the brain. Furthermore, although the level of injury varies among reported cases, no pattern fitting the vascular "watershed zones" of the spinal cord has been documented. ${ }^{27}$

\section{Clinical Presentations}

Clinical picture consists of an initial flaccid paraparesis or paraplegia of sudden onset with sensory loss in the legs and lower thoracic dermatomes and urinary sphincter disturbances. Occasionally the sensory and motor levels extend to the cervical region, ${ }^{31}$ resembling symptoms of transverse myelitis.

\section{Pathology Findings}

Extensive necrosis of the cervical and thoracic cord has been described in some patients. ${ }^{31}$

\section{Imaging Findings}

MRI is frequently normal. Hyperintense lesions on T2-WI and fluid attenuation inversion recovery (FLAIR) images affecting the posterior and lateral columns, pontomedullary region, and ventral pons may be seen. Findings consistent with acute transverse myelitis have also been reported. Contrast enhancement is variable and usually patchy. ${ }^{27}$

\section{Diagnosis}

Most reports describe normal CSF findings although pleocytosis and increased total protein may occur. ${ }^{28-30,32}$

\section{Treatment or Prognosis}

Treatment with either intravenous corticosteroids or immunopheresis may limit immune response preventing disability but their efficacy requires further validation. ${ }^{27}$ Prognosis is often poor, with residual spastic paraparesis and sensory deficits, and in some patients death. ${ }^{29}$ Full recovery has been reported occasionally. ${ }^{31}$

\section{Hepatic Myelopathy \\ Definition}

Hepatic myelopathy or portosystemic myelopathy is a rare neurologic complication of chronic liver disease with portal hypertension usually associated with portosystemic shunting either surgically created or spontaneous. It is characterized by an insidious onset of pure motor spastic paraparesis without sensory or sphincter involvement. ${ }^{33}$

\section{Pathophysiology}

Pathophysiology is poorly understood and it is broadly accepted that nitrogenous products such as ammonia, fatty acids, indoles, and mercaptans bypassing the liver through a portocaval shunt play an important role. ${ }^{34}$ These metabolites cause myelin damage resulting in demyelination in the brain and spinal cord. Selective predisposition of the motor system has been demonstrated by involvement of the lateral corticospinal tracts in autopsy studies. Other causative factors include nutrient deficiencies and deranged liver metabolism. ${ }^{33}$ Additionally, the myelopathy may also be related to elevated manganese levels with MRI evidence of manganese deposition in the basal ganglia without extrapyramidal manifestations or clinical evidence of decompensated cirrhosis. ${ }^{1,26,35}$

\section{Clinical Presentations}

The typical clinical presentation is a progressive pure motor spastic paraparesis with minimal or no sensory deficits and no bowel or bladder involvement. Most patients report prior episodes of hepatic encephalopathy and often the development of myelopathy follow the creation of surgical shunts. However, preceding portosystemic encephalopathy is not invariably present. ${ }^{1}$ Early and accurate diagnosis is important because patients with early stages of the disease can recover following liver transplantation. ${ }^{33,36}$

\section{Pathology Findings}

Neuropathologic studies show demyelination in the lateral corticospinal tracts with varying degrees of axonal loss. ${ }^{33,37}$

\section{Imaging Findings}

MRI usually shows symmetric high T2 signal intensity in the lateral corticospinal tracts and less commonly in the ventral pyramidal tracts, posterior columns, and spinocerebellar tracts. ${ }^{36}$ Increased pallidal Tl signal in presence of elevated serum manganese may also be present.

\section{Diagnosis}

Hepatic myelopathy is diagnosis of exclusion. Motor evoked potential studies may be suitable for the early diagnosis even in patients in the preclinical stages of the disease. ${ }^{33,38}$

\section{Treatment or Prognosis}

Hepatic myelopathy is a rare and debilitating disorder with no effective treatment. However, its early identification and exclusion of other treatable causes is important. Some patients improve following liver transplantation ${ }^{26}$ but the therapeutic potential of liver transplantation for preventing progression and allowing recovery needs to be further validated. ${ }^{3}$ 


\section{Organophosphate Poisoning Definition}

The most common complication of organophosphate poisoning is an acute intoxication with a cholinergic crisis because of the inhibition of acetylcholinesterase. Some patients subsequently develop organophosphate-induced delayed myelopathy and neuropathy, also known as organophosphate-induced delayed neurotoxicity (OPIDN).

\section{Pathophysiology \\ Organophosphates are common components of pesticides available in many homes. A major chemical that causes symptoms is triorthocresyl phosphate. Signs and symptoms of acute organophosphate toxicity are because of acetylcholi- nesterase inhibition and resulting muscarinic and nicotinic dysfunctions. In the neuromuscular junction, acetylcholine is released when a nerve impulse reaches terminal axonal end and it diffuses across the synaptic cleft and binds to cholinergic nicotinic receptors on the muscle fibers, causing them to contract. The enzyme, cholinesterase splits acetylcholine into acetic acid and choline, stopping its action. End products of the metabolism of acetylcholine are taken up by nerve fibers and resynthesized into acetylcholine. In organophosphate poison- ing, cholinesterases are phosphorylated by the phosphate end of organophosphates resulting in excessive accumulation of acetyl chlorine with resultant effects on muscarinic, nicotinic receptors in the CNS. ${ }^{39}$ After resolution of a cholinergic crisis, an intermediate syndrome can develop in some patients. It is likely a neuromuscular junction defect syndrome. ${ }^{40}$ OPIDN is a late complication of some organophosphorus compounds and is possibly because of phosphorylation and subsequent aging of neurotoxic esterase in the nervous system. ${ }^{1,41}$}

\section{Clinical Presentations}

After organophosphate poisoning, 3 well-defined clinical phases may be seen as follows: an initial acute cholinergic crisis, an intermediate syndrome, and OPIDPN. Pinpoint pupils, excessive salivation, generalized weakness, and respiratory collapse characterize the acute cholinergic crisis. The intermediate syndrome is characterized by weakness of neck flexors, proximal limbs, and respiratory muscles. OPIDN occurs 1-3 weeks after acute exposure and following an uncertain duration after chronic exposure. OPIDN may occur in absence of the cholinergic or intermediate phase. Symptoms include distal paresthesias, progressive leg weakness and wasting, and cramping muscle pain. There may be evidence of upper limb involvement and pyramidal tract dysfunction. Sensory loss when present is mild. ${ }^{1}$

\section{Pathology Findings}

Studies in chicks with organophosphate-induced delayed neuropathy show severe damage in the ventral and lateral tracts of the thoracic spinal cord. The same neuropathologic changes may be associated with prominent and diffuse spinal cord atrophy especially in the thoracic region. ${ }^{42}$

\section{Imaging Findings}

MRI at the late phase of exposure may reveal evidence of spinal cord atrophy that persists long after the cholinergic effects had subsided.

\section{Diagnosis}

There is usually a delay in onset of clinical symptoms after exposure to organophosphates. Measurement of cholinesterase activity in red blood cells helps confirm the diagnosis because red blood cells cholinesterase activity is less rapidly depressed than serum cholinesterase activity. ${ }^{1,26,43}$ In the late phase somatosensory evoked potentials are often abnormal.

\section{Treatment or Prognosis}

Acute symptoms are best treated with pralidoxime and atropine. Pralidoxime is a reactivator of inhibited acetylcholinesterase and is its specific antidote. ${ }^{1}$

\section{Chemotherapy-Related Myelopathies \\ Definition}

Acute myelopathy is a rare but devastating complication of chemotherapy treatment. It has been reported following administration of different chemotherapeutic agents including methotrexate, cytarabine, cisplatin, cladarabine, doxorubicin, vincristine, and cytosine arabinoside. ${ }^{26}$

\section{Pathophysiology}

Intrathecal methotrexate and cytarabine are the most frequently implicated agents. ${ }^{44}$ Acute or chronic leukoencephalopathy is the most common type of methotrexate-induced neurotoxicity and subacute myelopathy is rare. ${ }^{45}$ The pathogenesis of methotrexate-induced myelopathy is not clear but it is probably multifactorial; Following 2 major mechanisms have been proposed: indirect inhibition of methionine synthesis and direct toxicity. Methotrexate inhibits the enzyme dihydrofolate reductase and prevents folic acid from converting to tetrahydrofolic acid causing folate deficiency. This deficiency indirectly inhibits the synthesis of methionine, as folate is an important cofactor in DNA synthesis and methylation pathways. As methionine is necessary for formation and maintenance of myelin sheaths, its deficiency may lead to vacuolar degeneration of white matter. This mechanism is similar to that of subacute combined degeneration of the spinal cord because of vitamin $\mathrm{B}_{12}$ deficiency. A constitutional predisposition or abnormal sensitivity to methotrexate such as genetic polymorphisms or mutations related to methionine metabolism may also play a role in the pathogenesis as the reported frequency of severe methotrexate myelopathy is disproportionately low considering the large number of patients receiving the drug intrathecally. ${ }^{46}$ Another proposed mechanism is direct injury of endothelial cells in venules and capillaries of the spinal cord with blood-brain barrier disruption, increased vascular permeability, and insinuation of plasma proteins into the vascular walls ${ }^{46}$ which lead to their degeneration and necrosis and vacuolar degeneration of white matter. Vascular injury also allows the drug to diffuse into the CNS causing direct toxic effects. Direct toxic effect of intrathecal 
administration of methotrexate on the pia mater and white matter is probably caused by pia-glial barrier disruption and myelin sheaths and oligodendrocytes injuries. ${ }^{46}$

Known risk factors for development of methotrexate myelopathy include the following: cumulative intrathecal doses and continuous exposure to the drug more than a prolonged period of time, systemic administration, concurrent radiotherapy, and CNS involvement by the primary disease. ${ }^{47}$

\section{Clinical Presentations}

There is a variable clinical course in regard to time and rate of onset after therapy, severity of disease, and degree of recovery. ${ }^{48}$ Overall, 2 clinical patterns of paraparesis have been described. ${ }^{49}$ A transient, flaccid, and areflexic paraparesis with pain and anesthesia may occur soon after intrathecal injection. Rarely, the paralysis may ascend with respiratory compromise and death. A less common form is a progressive spastic-ataxic paraparesis with sphincter dysfunction seen after weeks of a series of intrathecal treatments. ${ }^{1}$ The latency to onset of symptoms is variable and can be long.

\section{Pathology Findings}

Reports on the neuropathologic findings of methotrexateinduced myelopathy are rare and findings vary from vacuolar degeneration of the lateral and posterior columns in association with severe loss of axons and myelin sheaths to complete transverse necrosis of the spinal cord or a combination of both. ${ }^{46}$ The gray matter is relatively spared. ${ }^{46}$

\section{Imaging Findings}

MRI findings are nonspecific and the initial imaging examination is often normal. Symmetric increased T2 signal in dorsal and lateral columns of the cord without abnormal contrast enhancement similar to that of subacute combine degeneration has been described (Fig. 6). Cord swelling and contrast enhancement have also been reported. Long-term outcome is generally that of cord atrophy with normal $\mathrm{T} 2$ signal intensity demonstrated as early as 6 months after clinical onset of disease. $^{48,50}$

\section{Diagnosis}

Chemotherapy-induced myelopathy is a diagnosis of exclusion. The main differential diagnosis includes malignancy, viruses, radiation, and paraneoplastic myelopathy. ${ }^{48}$

\section{Treatment or Prognosis}

Chemotherapy-induced myelopathy carries a poor prognosis. Preservative-containing chemotherapeutic agents or diluents should not be used intrathecally ${ }^{49}$ and multiple and frequent intrathecal therapy should be avoided. ${ }^{1}$ Meticulous neurologic monitoring of methotrexate concentration in CSF is important to prevent the occurrence of this complication. ${ }^{46}$ Immediate discontinuation of intrathecal methotrexate treatment and supplementation of derivates of folic acid and methionine or homocysteine metabolic pathway, such as S-adenosylmethionine and cyanocobalamine, are the best treatment options. ${ }^{51}$ However, further research is required to establish an effective and definite treatment. ${ }^{45}$

\section{Radiation-Induced Myelopathies Definition}

Radiation myelopathy is defined as injury caused by ionizing radiation. ${ }^{52}$ There are 2 main reasons for spinal cord exposure to radiation-an intentional delivery to treat primary or secondary lesions or its inevitable inclusion in a radiation field which intends to treat an adjacent neoplasm. In either case, factors such as total radiation dose, radiation dose per fraction, time between applications, spinal cord level and associated chemotherapy affect the incidence and severity of myelopathy. ${ }^{52}$ Radiation myelopathy is divided into the following 4 categories: acute transient radiation myelopathy, acute paraplegia or quadriplegia, lower motor neuron findings, and chronic progressive radiation myelopathy also known as delayed radiation myelopathy (DRM). DRM is rare but is the most feared complication and typically occurs after a certain latent period that ranges from months to few years following irradiation. ${ }^{26,52}$

\section{Pathophysiology}

The pathogenesis of DRM is likely to be multifactorial with no single target cell and no single pathway solely responsible. Damage to vascular endothelial cells, ${ }^{53}$ direct injury of oligodendroglia and myelin sheaths, ${ }^{54}$ and disturbance of the intra-spinal venous drainage because of lesions in the vein walls ${ }^{55}$ have been considered most important. ${ }^{46}$

It is known that the spinal cord has an enhanced radiosensitivity relative to other organs and that there is no uniformly accepted "spinal cord tolerance" and that no precise threshold dose is considered safe. ${ }^{56} \mathrm{~A}$ total dose of $45 \mathrm{~Gy}$ with fractions of $1.8-2 \mathrm{~Gy}$ is generally accepted as dose limit using conventional radiotherapy ${ }^{57}$ although 40 Gy poses however a small risk of myelopathy. Doses of $45 \mathrm{~Gy}$ and $52 \mathrm{~Gy}$ to the cervical cord result myelopathy rates of $0.1 \%$ and $1 \%$ respectively with a lower incidence in the thoracic cord. ${ }^{48,58}$ Reports of myelopathy after stereotactic radiosurgery to spinal lesions are rare. ${ }^{59}$

Concomitant chemotherapy is a recognized risk factor for development of radiation myelopathy. In the past years, targeted antibody and antireceptor therapies have emerged, but the extent to which these agents contributed to the development of radiation myelopathy remains unknown. ${ }^{57}$ Previous exposure to radiation, particularly less than 2 years, may also carry an increased risk as spinal cord damage is most likely to be become overt during this period. ${ }^{56}$ The volume and length of spinal cord as well as the spinal cord segment irradiated may also influence the incidence of radiation myelopathy. ${ }^{56}$

Other factors in the patients' medical history and physical status make the spinal cord sensitive to radiation injury. Hypertension, diabetes, autoimmune disease, congenital and acquired spinal cord abnormalities, pre-existing CNS injury, elevated hemoglobin, hypotension, alcohol abuse, and infectious disease also enhance the cord's sensitivity to radiation damage. ${ }^{60}$

\section{Clinical Presentations}

Overall, 4 clinical syndromes of radiation myelopathy have been described are as follows: (1) an acute transient radiation 


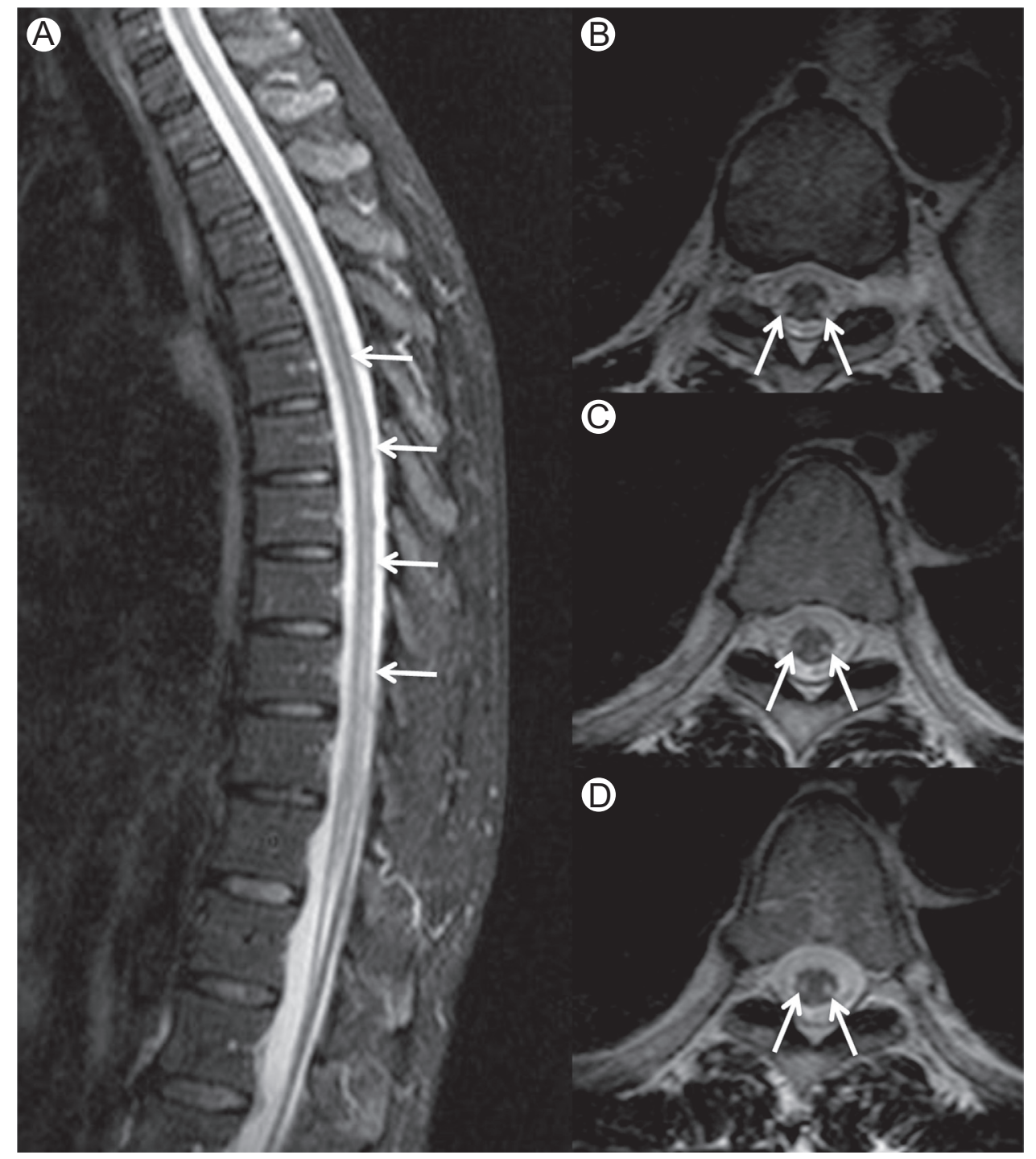

Figure 6 Methotrexate myelopathy. Midsagittal (A) and axial (B-D) STIR images in a patient who underwent intrathecal methotrexate therapy for acute lymphoblastic leukemia. Images show high-signal intensity in dorsal and lateral columns (arrows) mimicking subacute combined degeneration. STIR, short-tau inversion recovery. (Courtesy: Lazaro Amaral, Sao Paulo, Brazil.)

myelopathy (most common) characterized by Lhermitte's sign with no other abnormalities on neurologic examination, (2) an acutely developing paraplegia or quadriplegia presumably secondary to ischemia and hemorrhage of the spinal cord caused by radiation-induced blood vessel damage, (3) a lower motor neuron disease in the upper or lower extremities, presumably caused by selective anterior horn-cell damage, and (4) DRM. 52,56,61 Although the second and the third syndromes are exceedingly rare, the last one is the one most concerning.

Acute transient radiation myelopathy is a self-limiting early delayed reaction usually treated with corticosteroids. It occurs after a latent period of 2-4 months and is characterized by brief electric-like sensations in the spine and extremities triggered by neck flexion or extension (Lhermitte's sign). Clinical improvement is generally seen within 2-9 months followed by complete recovery. There is no correlation between acute transient radiation myelopathy and DRM. Conversely DRM is a devastating condition, which may be permanent. It generally occurs after a latent period of time of 9-18 months but has been reported up to 3-4 years after completion of radiation treatment. Various combinations of motor and sensory deficits occur and depend on the anatomic level of cord injury. Development of bowel and bladder dysfunctions may occur. DRM may be fatal if it involves the upper cervical level.

\section{Pathology Findings}

Myelinated fibers and blood vessels are the most affected structures. In chronic progressive radiation myelopathy white matter necrosis and demyelination with vascular lesions including stenosis, thrombosis, hemorrhage, fibrinoid vascular necrosis, and venous wall thickening and hyalinization are seen. ${ }^{62}$ There is also gray matter involvement, including 
astrocytosis and hyalinosis of the vessel walls, but neurons are usually spared. ${ }^{60}$

\section{Imaging Findings}

In acute transient radiation myelopathy MRI is usually normal. In DRM, MRI shows cord swelling initially and a long intramedullary lesion with increased T2 signal and contrast enhancement (Fig. 7). The MRI findings correlate with neurologic symptoms. In the chronic stage MRI demonstrates cord atrophy. Radiation-induced bone marrow changes indicate the extension of the irradiated field and may help in reaching the correct diagnosis.

Positron emission tomography-computed tomography studies might have a role in differentiating radiation myelopathy from primary spinal cord lesions and spinal metastases. However, very few positron emission tomography-computed tomography studies have been conducted in the spinal cord and their results need validation. ${ }^{52}$

\section{Diagnosis}

Diagnostic criteria for DRM include-(1) the affected spinal cord segment must be in the irradiated zone, (2) symptomatology must correspond to the radiated spinal cord segment, (3) and the latency period should be more than 6 months. The differential diagnosis represents a challenging problem. Metastatic tumor is the most common cause of cord lesions in oncologic patients presenting with myelopathy. ${ }^{52}$ Primary intramedullary neoplastic lesions and other neurologic pathologies presenting with myelopathy must also be ruled out including paraneoplastic syndrome, zoster myelopathy, and myelopathy secondary to chemotherapy. ${ }^{52}$ Nerve conduction studies show slowing or complete block in spinal conduction. CSF examination is usually normal but mild elevation of protein levels and lymphocytosis may be seen. ${ }^{52,63,64}$

\section{Treatment or Prognosis}

No treatment has conclusively been shown to be of value in DRM. Several therapies such as corticosteroids, anticoagulation, pentoxifylline, vitamin E, and hyperbaric oxygen have been tried with limited benefits. Some patients derive a shortterm benefit from steroids which may be related to decreasing edema and inflammation. ${ }^{52}$

\section{Toxic Myelopathies Associated With Geographical Predilection}

\section{Spinal Sea Stroke \\ Definition}

Spinal sea stroke is characterized by spinal and brainstem infarctions that occur in patients swimming in the North Carolina and Virginia coasts probably caused by contact with toxic marine animals.
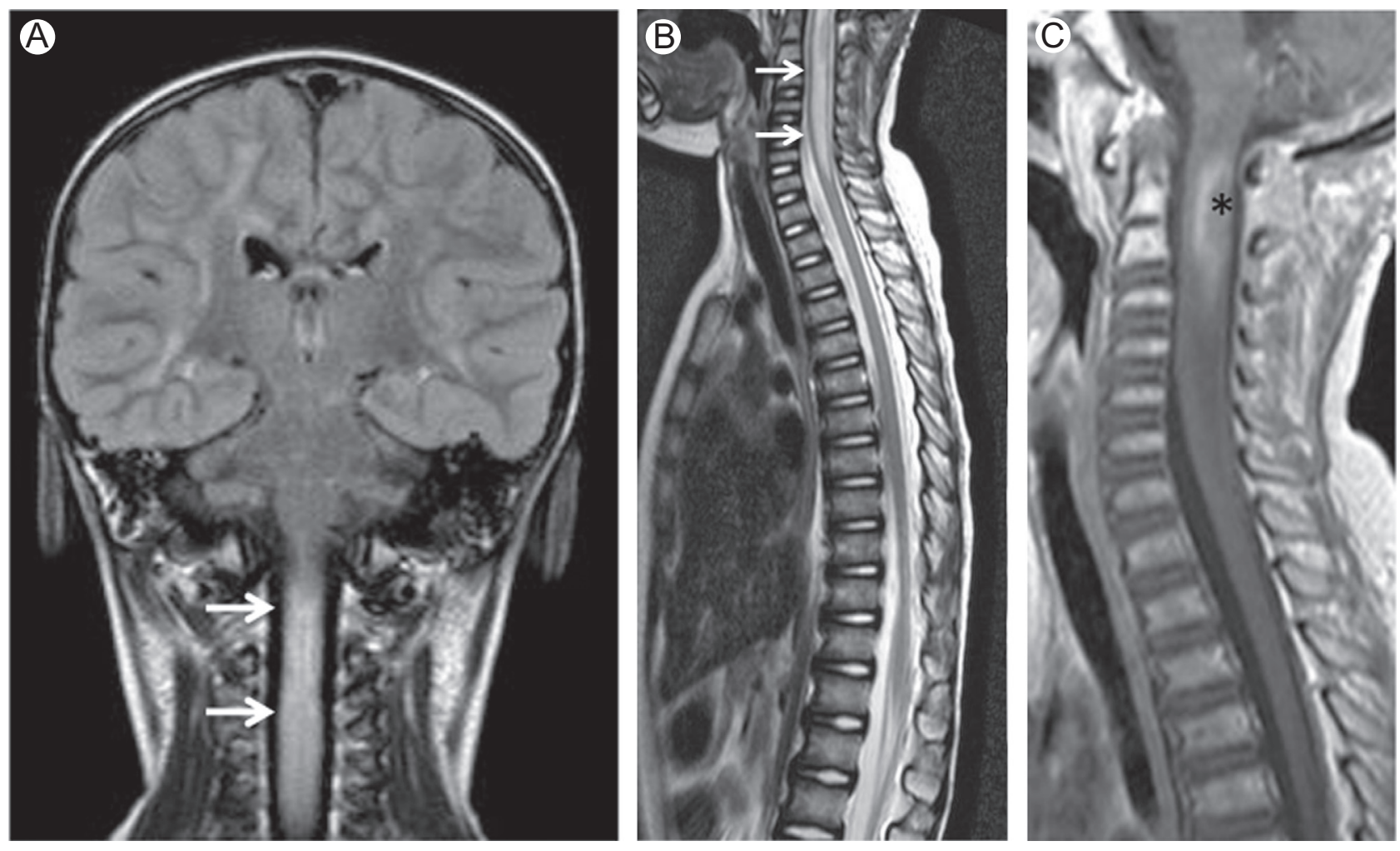

Figure 7 Radiation therapy-related myelopathy. Brain MRI coronal FLAIR (A), spine MRI midsagittal T2 (B) and midsagittal postcontrast T1-WI (C) of a patient who underwent radiation therapy. Radiation-induced myelopathy is seen as an intramedullary area of high-signal intensity on FLAIR (arrows on A) and T2-WI (arrows on B) with mild enhancement after gadolinium administration (* on C). FLAIR, fluid attenuation inversion recovery. (Courtesy: Dr Quaghebeur Oxford, UK.) 


\section{Pathophysiology}

Brainstem and spinal cord strokes in young adults are extremely rare. Jellyfish toxins have been proposed as a cause but the mechanism of neural tissue damage is unknown. It is also unclear why in all of the reported cases the abnormalities involved only the spinal cord and brainstem but spared the brain $^{65}$ Some jellyfish toxins affect the calcium channels of neurons, a process that could lead to cytotoxic edema, failure of cellular energetics, and eventually to irreversible damage such as seen in ischemia. Some authors have postulated that damage to the spinal cord is not direct but due to systemic hypotension with subsequent infarctions. ${ }^{65}$

\section{Clinical Presentations}

Patients typically present with an acute severe dysfunction of sensory and motor systems. Neurologic signs and symptoms depend on the level of spinal cord involvement and include severe back pain, loss of sphincter control, paraplegia or paraparesis, or quadriplegic or tetraplegia in patients with higher cord lesions. ${ }^{65}$

\section{Pathology Findings}

None of the published cases of spinal sea stroke report pathology findings.

\section{Imaging Findings}

The hallmark of spinal cord infarction is high T2 signal intensity in the cord (Fig. 8) especially involving the anterior two-third of the cord that correspond to the territory supplied by the anterior spinal artery. Diffusion weighted imaging shows restricted diffusion of the lesion.

\section{Diagnosis}

The diagnosis is generally made clinically. MRI should be obtained in all patients with suspected spinal cord infarction to confirm the diagnosis and to exclude other possible treatable causes of myelopathy.

\section{Treatment or Prognosis}

Spinal cord infarctions have a poor prognosis with little chance of recovery and significant sequelae.

\section{Subacute Myelo-Optico Neuropathy (Clioquinol Toxicity)}

Clioquinol (iodochlorhydroxyquin) is an antifungalantiprotozoal drug used to treat intestinal parasitic diseases. Clioquinol's use has been restricted or discontinued in some countries because of events in Japan (from 1955-1970) where more than 10,000 people developed a subacute myelo-optic neuropathy $(\mathrm{SMON})$. SMON is characterized by subacute onset of lower limb paresthesias and spastic paraparesis with optic atrophy. Examination findings include hyperreflexia and extensor plantar responses. The physiopathology mechanism is unknown but clioquinol is a copper or zinc chelator and this has led to the speculation that clioquinol-induced neurotoxicity may be a consequence of copper deficiency. ${ }^{1,26,66-68}$

\section{Lathyrism (India, Bangladesh, and Ethiopia) and Konzo (Africa)}

Lathyrism is a self-limiting neurotoxic disorder endemic in Bangladesh, India, and Ethiopia, caused by beta-N-oxalylamino-L-alanine, an excitotoxic amino acid present in Lathyrus sativus. Ingestion of this neurotoxin results in increased intracellular levels of reactive oxygen species and subsequent impairment of the mitochondrial oxidative phosphorylation chain. It presents as a subacute or insidious onset spastic paraparesis. Degeneration is most prominent in motor cortex and pyramidal tracts that are responsible for lower extremity function. ${ }^{1}$

Konzo is a tropical myelopathy seen in Africa resulting from weeks of high dietary cyanide exposure due to consumption of insufficiently processed cassava (Manihot esculenta Crantz) that contains cyanide. It is characterized by the abrupt onset of spastic non-progressive paraparesis. ${ }^{69}$ Years of cassava consumption have also been implicated in a syndrome of slowly progressive ataxia, peripheral neuropathy, and optic atrophy seen in parts of Africa. ${ }^{1,70}$ The diagnosis is based on a history of exposure and measurement of serum thiocyanate levels.

Lathyrism and Konzo are irreversible and non-progressive conditions. MRI studies are negative. Somatosensory evoked potentials may show abnormalities which indicate poor conduction through the spinal cord. ${ }^{26,71}$ There are no specific treatments for these entities.

\section{Other Noninfectious and Noninflammatory Myelopathies}

\section{Ketoacidosis Myelopathy \\ Definition}

Myelopathy is a rare complication of diabetic ketoacidosis characterized by spinal cord edema and infarction usually associated with cerebral findings.

\section{Pathophysiology}

There are no existing explanations for the spinal cord edema associated with diabetic ketoacidosis probably due its rarity. Current explanations are limited to diabetic children with cerebral edema but the pathophysiological mechanisms for cerebral edema and infarctions are not clear. Systemic hypotension and hypoxia, rapid changes in serum osmolality, disseminated intravascular coagulation, and thrombosis secondary to dehydration; hemoconcentration and hyperviscosity have all been proposed as possible causes. ${ }^{72-75}$ 

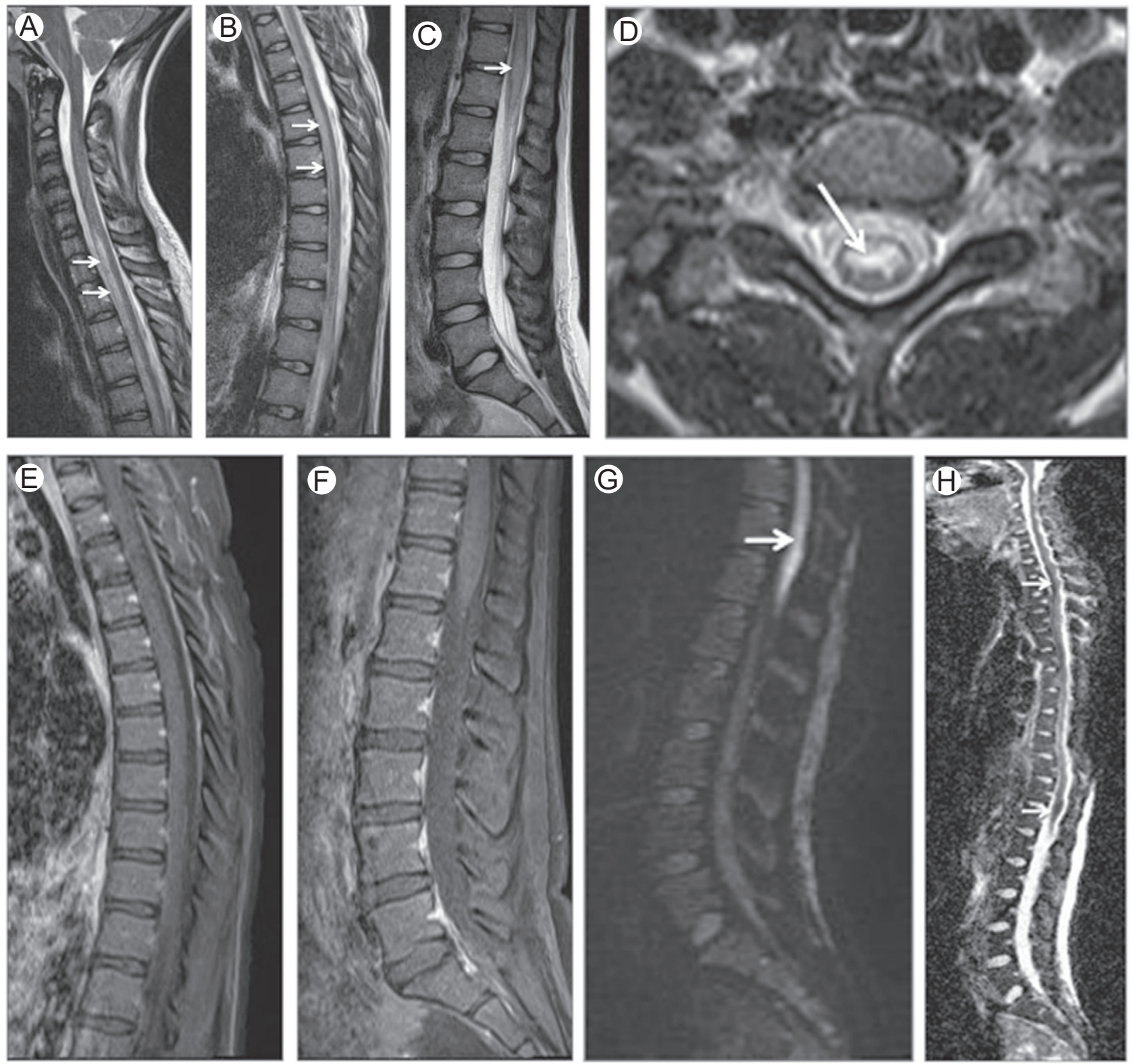

Figure 8 Spinal sea stroke. Sagittal T2-WI at the level of the cervical (A), thoracic (B), and lumbar (C) spine and axial T2-WI (D) show high T2-WI signal intensity in the anterior two-third of the cord. Sagittal postcontrast fat-suppressed T1-WI (E and F) show no contrast enhancement in areas of T2 signal abnormality. DWI (G) and ADC map (H) show restricted diffusion suggesting infarct. ADC, apparent diffusion coefficient.

\section{Clinical Presentations}

Clinical presentation depends on the affected spinal cord level.

\section{Pathology Findings}

No neuropathologic findings have been described in reported cases.

\section{Imaging Findings}

Cord edema is seen as high T2 signal in the spinal cord usually associated with cord expansion (Fig. 9). Spinal cord infarction is seen as areas of bright T2 signal that show restricted diffusion. Patchy enhancement after gadolinium administration has been reported. Atrophy may be seen in late stages.

\section{Diagnosis}

Ketoacidosis myelopathy is a diagnostic of exclusion.

\section{Treatment or Prognosis}

There is no effective treatment. The prognosis is poor and varies according with the severity of the disease. Cardiac failure and pulmonary edema are rare but possible complications in critically ill patients with diabetic ketoacidosis.

\section{Spinal Cord Decompression Illness}

\section{Definition}

Decompression sickness is a clinical syndrome caused by alterations in environmental pressure resulting in liberation of 
inert gas bubbles in tissues or blood. Sport divers generally use compressed air as their breathing mixture and the inert gas that is liberated during decompression is nitrogen. There are following 2 types of decompression illness: type I is characterized by joint pain, skin marbling, small patchy hemorrhage, and lymphatic obstruction and type II is characterized by CNS involvement. CNS involvement may be further subdivided in cerebral and spinal cord subtypes. Spinal cord involvement accounts for $77 \%$ of CNS diving injuries. The population at risk includes, in addition to sport divers, naval and commercial divers, aviators, astronauts, and compressed air workers.

\section{Pathophysiology}

The pathophysiological mechanism of spinal cord decompression is not totally understood. Hypotheses include embolization of arterial bubbles via a right-to-left shunt, development of bubbles within the spinal cord, or embolization of the epidural vertebral venous system causing congestion and venous cord infarctions. The last hypothesis is the best documented one as the alteration of spinal cord venous draining resulting from the obstruction of the epidural venous system has been shown experimentally. ${ }^{76}$ Secondary immunoinflammatory processes may contribute to worsening of symptoms. Nitrogen bubbles lodge in spinal veins at the thoracic level; there the spine mobility is restricted by the rib cage.

\section{Clinical Presentations}

The great majority of spinal cord decompression sickness results in subjective sensory abnormalities distributed according to the dermatomes. A less frequent but more characteristic presentation corresponds to numbness in the limbs with a progressive ascending level of both sensory and motor deficits often accompanied by disturbances of bladder function. ${ }^{77,78}$

\section{Pathology findings}

Spinal cord lesions are scattered throughout the white matter with no segmental distribution. The dorsolateral white matter is more vulnerable because of its high fat content in which nitrogen is readily stored.

\section{Imaging Findings}

MRI is reliable in the early detection of spinal cord lesions that occur in decompression accidents leading to prompt hyperbaric treatment and in the follow-up during and after hyperbaric recompression. MRI may demonstrate patchy areas of increased T2 signal intensity in the white matter tracts (Fig. 10).

\section{Diagnosis}

The diagnosis relies on clinical history, physical examination, and MRI findings.
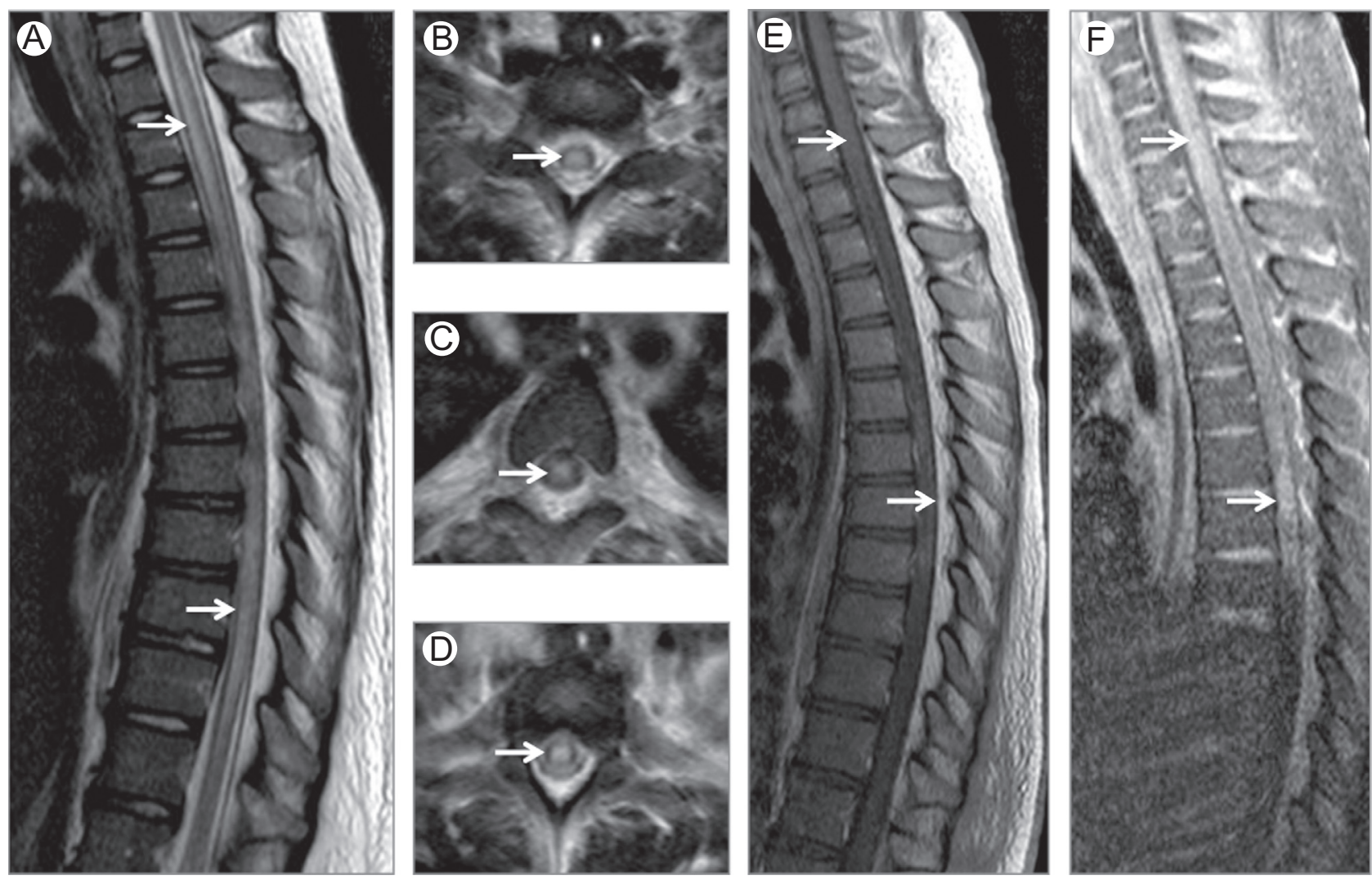

Figure 9 Ketoacidosis myelopathy. Sagittal (A) and axial (B-D) T2-WI of thoracic spine show high T2-WI signal intensity involving the central aspect of the cord (arrows). Sagittal T1-WI (E) and postcontrast fat sat T1-WI (F) show areas of contrast enhancement. 


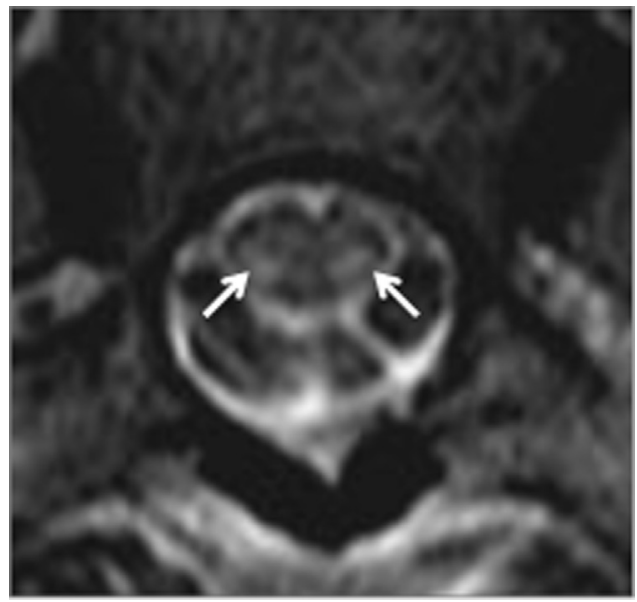

Figure 10 Spinal cord decompression sickness. Axial T2-WI at level of thoracic spine shows high-signal intensity in the dorsolateral white matter tracts. The lesions are usually scattered throughout the white matter with no segmental distribution. (Courtesy: A. Vidal, Santiago, Chile.)

\section{Treatment or Prognosis}

Hyperbaric oxygen therapy is the treatment of choice as it reduces the bubble volume and increases concentration of dissolved oxygen in ischemic tissues. Incomplete recovery is seen in 20\%-30\% of patients. ${ }^{79,80}$ Age, depth, bladder dysfunction, persistence or worsening of signs before recompression, and a Boussuges score more than 7 are independent predictive variables for poor clinical recovery. ${ }^{78}$

\section{Surfer's Myelopathy \\ Definition}

Surfer's myelopathy is a rare non-traumatic injury that occurs in novice surfers who lay prone on a surfboard with the lumbar spine hyperextended for prolonged periods of time. It is probably underdiagnosed and underreported as it is a rare condition only recently recognized. ${ }^{81}$

\section{Pathophysiology}

The pathophysiology of surfer's myelopathy is unknown. Arterial insufficiency from hyperextension of the lumbar spine, venous hypertension associated with obstruction of the inferior vena cava by the liver whereas lying in a prone position, fibrocartilaginous embolism caused by retrograde embolism of the nucleus pulpous into the radicular spinal cord arteries, or avulsion of perforating arteries have all been proposed. ${ }^{81,82}$

\section{Clinical Presentations}

Surfer's myelopathy is usually preceded by back discomfort followed by acute myelopathy with variable motor-sensory involvement and urinary incontinence.

\section{Pathology Findings}

No definite histopathologic confirmation has been published, but surfer's myelopathy probably is the result of a vascular phenomenon involving dynamic compression, vasospasm, or thrombotic infarction of the artery of Adamkiewicz that occurs as the result of hyperextension of the spinal cord whereas individuals are lying prone on a surfboard. .1,82 $^{2}$

\section{Imaging Findings}

Surfer's myelopathy is usually associated with a longitudinally extensive T2 hyperintense central cord lesion in the midthoracic region with variable cranial and caudal extension. Patchy increased $\mathrm{Tl}$ signal presumably related to petechial hemorrhage have been described. ${ }^{83}$ Enhancement of the ventral roots of the cauda equina have also reported. ${ }^{81,82,84,85}$

\section{Diagnosis}

The characteristic clinical history associated with the longitudinally extensive T2 hyperintense spinal cord lesion on MRI suggests the diagnosis.

\section{Treatment or Prognosis}

No treatment is available. Recovery is variable ranging from complete recovery of function to persistent paraplegia.

\section{Conclusion}

Toxic and metabolic myelopathies and myeloneuropathies are relatively rare clinical conditions that share clinical, neuropathologic, and imaging features. Acquaintance with these entities may lead to accurate diagnosis with successful identification of the underlying cause and appropriate treatment which may revert or prevent progression of symptoms associated with these incapacitating disorders.

\section{References}

1. Kumar N: Metabolic and toxic myelopathies. Semin Neurol 32(2): 123-136, 2012

2. Timms SR, Curé JK, Kurent JE: Subacute combined degeneration of the spinal cord: MR findings. AJNR Am J Neuroradiol 14(5):1224-1227, 1993

3. Goodman BP: Metabolic and toxic causes of myelopathy. Continuum (Minneap Minn) 21:84-99, 2015. [1 spinal cord disorders]

4. Katsaros VK, Glocker FX, Hemmer B, et al: MRI of spinal cord and brain lesions in subacute combined degeneration. Neuroradiology 40(11): 716-719, 1998

5. Sotirchos ES, Saidha S, Becker D: Neurological picture. Nitrous oxideinduced myelopathy with inverted V-sign on spinal MRI. J Neurol Neurosurg Psychiatr 83(9):915-916, 2012

6. Ravina B, Loevner LA, Bank W: MR findings in subacute combined degeneration of the spinal cord: A case of reversible cervical myelopathy. AJR Am J Roentgenol 174(3):863-865, 2000

7. Jain KK, Malhotra HS, Garg RK, et al: Prevalence of MR imaging abnormalities in vitamin B12 deficiency patients presenting with clinical features of subacute combined degeneration of the spinal cord. J Neurol Sci 342(1-2):162-166, 2014

8. Küker W, Thron A: Subacute combined degeneration of the spinal cord: Demonstration of contrast enhancement. Neuroradiology 41(5):387, 1999

9. Lim CCT: Neuroimaging in postinfectious demyelination and nutritional disorders of the central nervous system. Neuroimaging Clin N Am 21(4): 843-858, 2011. [viii]

10. Su S, Libman RB, Diamond A, et al: Infratentorial and supratentorial leukoencephalopathy associated with vitamin B12deficiency. J Stroke Cerebrovasc Dis 9(3):136-138, 2000

11. Pacheco FT, Scoppetta TLPD, Sznirer M, et al: Acute demyelination with leukoencephalopathy and cerebellar involvement in vitamin B12 deficiency. Arq Neuropsiquiatr 73(3):257, 2015 
12. Renard D, Dutray A, Remy A, et al: Subacute combined degeneration of the spinal cord caused by nitrous oxide anaesthesia. Neurol Sci 30(1): 75-76, 2009

13. Ernst LD, Brock K, Barraza LH, et al: Longitudinally extensive nitrous oxide myelopathy with novel radiographic features. J Am Med Assoc Neurol 72(11):1370-1371, 2015

14. Botez MI, Reynolds EH, eds. Folic acid in neurology, psychiatry and internal medicine. New York: Raven Press, 1-5, 1979

15. Kumar N, Ahlskog JE, Klein CJ, et al: Imaging features of copper deficiency myelopathy: A study of 25 cases. Neuroradiology 48(2): 78-83, 2006

16. Kumar N: Copper deficiency myelopathy (human swayback). Mayo Clin Proc 81(10):1371-1384, 2015

17. Page PS, Nazar RG, Park MC, et al: Copper deficiency myelopathy in the setting of advanced degenerative cervical spondylosis. Br J Neurosurg:1-3, 2015

18. Silva-Júnior FPD, Machado AAC, Lucato LT, et al: Resolution of MRI findings of copper deficiency myeloneuropathy in a patient with Wilson's disease. Arq Neuropsiquiatr 72(3):255-256, 2014

19. Nagappa M, Bindu PS, Adwani S, et al: Clinical, hematological, and imaging observations in a 25-year-old woman with abetalipoproteinemia. Ann Indian Acad Neurol 17(1):113-116, 2014

20. Nelson JS: Neuropathological studies of chronic vitamin E deficiency in mammals including humans. Ciba Found. Symp 101:92-105, 1983

21. Vorgerd M, Tegenthoff M, Kühne D, et al: Spinal MRI in progressive myeloneuropathy associated with vitamin E deficiency. Neuroradiology 38(suppl 1):S111-S113, 1996

22. Müller KI, Bekkelund SI: Epilepsy in a patient with ataxia caused by vitamin E deficiency. Br Med J Case Rep 2011: 2011

23. Bilgrami M, O'Keefe P: Neurologic diseases in HIV-infected patients. Handb Clin Neurol 121:1321-1344, 2014

24. Tan SV, Guiloff RJ: Hypothesis on the pathogenesis of vacuolar myelopathy, dementia, and peripheral neuropathy in AIDS. J Neurol Neurosurg Psychiatr 65(1):23-28, 1998

25. Bizaare M, Dawood H, Moodley A: Vacuolar myelopathy: A case report of functional, clinical, and radiological improvement after highly active antiretroviral therapy. Int J Infect Dis 12(4):442-444, 2008

26. Schwendimann RN: Metabolic, nutritional, and toxic myelopathies. Neurol Clin 31(1):207-218, 2013

27. Haghi-Ashtiani B, Sina F, Ben Isa F, et al: Transverse myelitis and heroin abuse: A case report. Med J Islam Repub Iran 23(4):238-240, 2010

28. Richter RW, Rosenberg RN: Transverse myelitis associated with heroin addiction. J Am Med Assoc 206(6):1255-1257, 1968

29. Ell JJ, Uttley D, Silver JR: Acute myelopathy in association with heroin addiction. J Neurol Neurosurg Psychiatr 44(5):448-450, 1981

30. Grassa C, Montanari E, Scaglioni A, et al: Acute heroin myelopathy-Case report. Ital J Neurol Sci 5(1):63-66, 1984

31. Goodhart LC, Loizou LA, Anderson M: Heroin myelopathy. J Neurol Neurosurg Psychiatr 45(6):562-563, 1982

32. Lee MC, Randa DC, Gold LH: Transverse myelopathy following the use of heroin. Minn Med 59(2):82-83, 1976

33. Premkumar M, Bagchi A, Kapoor N, et al: Hepatic myelopathy in a patient with decompensated alcoholic cirrhosis and portal colopathy. Case Reports Hepatol 2012(4):1-4, 2012

34. Malhotra HS, Paliwal VK, Singh MK: Hepatic myelopathy: An unusual complication of advanced hepatic disease. Ann Neurosci 14(1), 2007

35. Gospe SM, Caruso RD, Clegg MS, et al: Paraparesis, hypermanganesaemia, and polycythaemia: A novel presentation of cirrhosis. Arch Dis Child 83(5):439-442, 2000

36. Weissenborn K, Tietge UJF, Bokemeyer M, et al: Liver transplantation improves hepatic myelopathy: Evidence by three cases. Gastroenterology 124(2):346-351, 2003

37. Zieve L, Mendelson DF, Goepfert M: Shunt encephalomyelopathy. II. Occurrence of permanent myelopathy. Ann Intern Med 53:53-63, 1960

38. Nardone R, Buratti T, Oliviero A, et al: Corticospinal involvement in patients with a portosystemic shunt due to liver cirrhosis: A MEP study. J Neurol 253(1):81-85, 2006

39. Azazh A: Severe organophosphate poisoning with delayed cholinergic crisis, intermediate syndrome and organophosphate induced delayed polyneuropathy on succession. Ethiop J Health Sci 21(3):203-208, 2011

40. Senanayake N, Karalliedde L: Neurotoxic effects of organophosphorus insecticides. An intermediate syndrome. N Engl J Med 316(13):761-763, 1987

41. Lotti M, Becker CE, Aminoff MJ: Organophosphate polyneuropathy: Pathogenesis and prevention. Neurology 34(5):658-662, 1984

42. Funk KA, Henderson JD, Liu CH, et al: Neuropathology of organophosphate-induced delayed neuropathy (OPIDN) in young chicks. Arch Toxicol 68(5):308-316, 1994

43. Chuang C-C, Lin T-S, Tsai M-C: Delayed neuropathy and myelopathy after organophosphate intoxication. N Engl J Med 347(14):1119-1121, 2002

44. Watterson J, Toogood I, Nieder M, et al: Excessive spinal cord toxicity from intensive central nervous system-directed therapies. Cancer 74(11): 3034-3041, 1994

45. Yi Y, Kang HJ, Shin HY, et al: Progressive myelopathy mimicking subacute combined degeneration after intrathecal chemotherapy. J Child Neurol 30(2):246-249, 2015

46. Shintaku M, Toyooka N, Koyama T, et al: Methotrexate myelopathy with extensive transverse necrosis: Report of an autopsy case. Neuropathology 34(6):547-553, 2014

47. Gosavi T, Diong CP, Lim S-H: Methotrexate-induced myelopathy mimicking subacute combined degeneration of the spinal cord. J Clin Neurosci 20(7):1025-1026, 2013

48. Counsel P, Khangure M: Myelopathy due to intrathecal chemotherapy: Magnetic resonance imaging findings. Clin Radiol 62(2):172-176, 2007

49. Hahn AF, Feasby TE, Gilbert JJ: Paraparesis following intrathecal chemotherapy. Neurology 33(8):1032-1038, 1983

50. Lu C-H, Yao M, Liu H-M, et al: MR findings of intrathecal chemotherapyrelated myelopathy in two cases: Mimicker of subacute combined degeneration. J Neuroimaging 17(2):184-187, 2007

51. Schlegel U: Central nervous system toxicity of chemotherapy. Eur Assoc NeuroOncol Mag 1(1):25-29, 2001

52. Kadir T, Sarica FB, Ozgur K, et al: Delayed radiation myelopathy: Differential diagnosis with positron emission tomography/computed tomography examination. Asian J Neurosurg 7(4):206-209, 2012

53. Jellinger K, Sturm KW: Delayed radiation myelopathy in man. Report of twelve necropsy cases. J Neurol Sci 14(4):389-408, 1971

54. Burns RJ, Jones AN, Robertson JS: Pathology of radiation myelopathy. J Neurol Neurosurg Psychiatr 35(6):888-898, 1972

55. Okeda R: Two autopsy cases of radiation myelopathy. Consideration about the pathogenesis. Shinkei Kenkyu No Shinpo 15(3):619-639, 1971

56. Gocheva L: Radiation tolerance of the spinal cord: Doctrine, dogmas, data. Arch Oncol:1-4, 2000

57. Gibbs IC, Patil C, Gerszten PC, et al: Delayed radiation-induced myelopathy after spinal radiosurgery. Neurosurgery 64(suppl):A67-A72, 2009

58. Werner-Wasik M, Yu X, Marks LB, et al: Normal-tissue toxicities of thoracic radiation therapy: Esophagus, lung, and spinal cord as organs at risk. Hematol Oncol Clin North Am. 18(1):131-160, 2004. [x-xi]

59. Pompili A, Crispo F, Raus L, et al: Symptomatic spinal cord necrosis after irradiation for vertebral metastatic breast cancer. J Clin Oncol 29(3):e53-6, 2011

60. Koehler PJ, Verbiest H, Jager J, et al: Delayed radiation myelopathy: Serial MR-imaging and pathology. Clin Neurol Neurosurg 98(2):197-201, 1996

61. Bowen J, Gregory R, Squier M, et al: The Post-Irradiation Lower Motor Neuron Syndrome Neuronopathy or Radiculopathy? Oxford University Press, 1996, http://dx.doi.org/10.1093/brain/119.5.1429

62. Soussain C, Ricard D, Fike JR, et al: CNS complications of radiotherapy and chemotherapy. Lancet 374(9701):1639-1651, 2009

63. Yasui T, Yagura H, Komiyama M, et al: Significance of gadoliniumenhanced magnetic resonance imaging in differentiating spinal cord radiation myelopathy from tumor: Case report. J Neurosurg 77(4): 628-631, 1992

64. Chao MW, Wirth A, Ryan G, et al: Radiation myelopathy following transplantation and radiotherapy for non-Hodgkin's lymphoma. Int J Radiat Oncol Biol Phys 41(5):1057-1061, 1998 
65. Chiang F, Castillo M: Seastrokes: A new threat for North Carolina swimmers? Neuroradiol J 27:499-502, 2014

66. Schaumburg H, Herskovitz S: Copper deficiency myeloneuropathy: A clue to clioquinol-induced subacute myelo-optic neuropathy? Neurology 71(9):622-623, 2008

67. Mao X, Schimmer AD: The toxicology of Clioquinol. Toxicol Lett 182(1): $1-6,2008$

68. Konagaya M, Matsumoto A, Takase S, et al: Clinical analysis of longstanding subacute myelo-optico-neuropathy: Sequelae of clioquinol at 32 years after its ban. J Neurol Sci 218(1-2):85-90, 2004

69. Howlett WP, Brubaker GR, Mlingi N, et al: Konzo, an epidemic upper motor neuron disease studied in Tanzania. Brain. 113(Pt 1):223-235, 1990

70. Osuntokun BO: An ataxic neuropathy in Nigeria. A clinical, biochemical and electrophysiological study. Brain 91(2):215-248, 1968

71. Nzwalo H, Cliff J: Konzo: From poverty, cassava, and cyanogen intake to toxico-nutritional neurological disease. PLoS Negl Trop Dis 5(6):e1051, 2011

72. Dixon AN, Jude EB, Banerjee AK, et al: Simultaneous pulmonary and cerebral oedema, and multiple CNS infarctions as complications of diabetic ketoacidosis: A case report. Diabet Med 23(5):571-573, 2006

73. Timperley WR, Preston FE, Ward JD: Cerebral intravascular coagulation in diabetic ketoacidosis. Lancet 1(7864):952-956, 1974

74. Roe TF, Crawford TO, Huff KR, et al: Brain infarction in children with diabetic ketoacidosis. J Diabetes Complications 10(2):100-108, 1996

75. Christodoulidou M, Selmi F: Severe diabetic ketoacidosis leading to cardiac failure, pulmonary oedema and spinal cord oedema resulting in tetraplegia. Br Med J Case Rep 2012, 2012

76. Hallenbeck JM: Cinephotomicrography of dog spinal vessels during corddamaging decompression sickness. Neurology 26(2):190-199, 1976
77. Gempp E, Blatteau J-E: Risk factors and treatment outcome in scuba divers with spinal cord decompression sickness. J Crit Care 25(2): 236-242, 2010

78. Blatteau J-E, Gempp E, Simon O, et al: Prognostic factors of spinal cord decompression sickness in recreational diving: Retrospective and multicentric analysis of 279 cases. Neurocrit Care 15(1): $120-127,2010$

79. Divers Alert Network: The DAN annual review of recreational SCUBA diving injuries and fatalities based on 1999 data. Report on Decompression Illness, Diving Fatalities and Project Dive Exploration. Durham, Divers Alert Network, 2001.

80. Divers Alert Network. The DAN annual review of recreational SCUBA diving injuries and fatalities based on 2003 data. Report on Decompression Illness, Diving Fatalities and Project Dive Exploration. Durham, Divers Alert Network, 2005.

81. Nakamoto BK, Siu AM, Hashiba KA, et al: Surfer's Myelopathy: A Radiologic Study of 23 Cases. American Journal of Neuroradiology. 34(12):2393-2398, 2013

82. Chang C, Donovan DJ, Liem LK, et al: Surfers' myelopathy. A case series of 19 novice surfers with nontraumatic myelopathy. Neurology 79(22): 2171-2176, 2012

83. Avilés-Hernández I, García-Zozaya I, DeVillasante JM: Nontraumatic myelopathy associated with surfing. J Spinal Cord Med 30(3):288-293, 2007

84. Thompson TP, Pearce J, Chang G, et al: Surfer's myelopathy. Spine (Phila Pa 1976) 29(16):E353-E356, 2004

85. Dhaliwal PPS, Cenic A, Eesa M, et al: An unusual case of myelopathy: Surfer's myelopathy. Can J Neurol Sci 38(2):354-356, 2011 